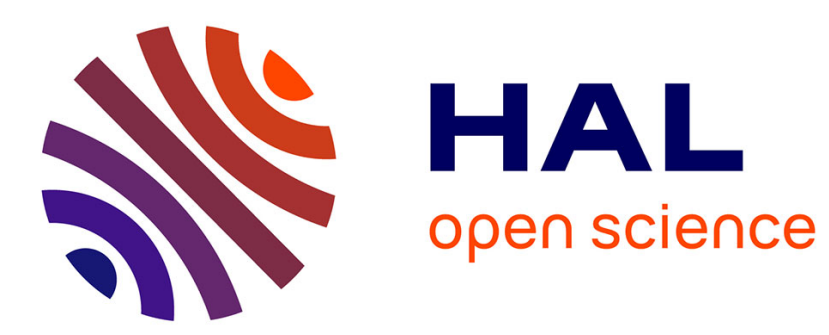

\title{
Élargissement et déplacement des raies de résonance magnétique causés par une excitation optique
}

\author{
Jean-Pierre Barrat, C. Cohen-Tannoudji
}

\section{To cite this version:}

Jean-Pierre Barrat, C. Cohen-Tannoudji. Élargissement et déplacement des raies de résonance magnétique causés par une excitation optique. J. Phys. Radium, 1961, 22 (7), pp.443-450. 10.1051/jphysrad:01961002207044300 . jpa-00236476

\section{HAL Id: jpa-00236476 https://hal.science/jpa-00236476}

Submitted on 1 Jan 1961

HAL is a multi-disciplinary open access archive for the deposit and dissemination of scientific research documents, whether they are published or not. The documents may come from teaching and research institutions in France or abroad, or from public or private research centers.
L'archive ouverte pluridisciplinaire HAL, est destinée au dépôt et à la diffusion de documents scientifiques de niveau recherche, publiés ou non, émanant des établissements d'enseignement et de recherche français ou étrangers, des laboratoires publics ou privés. 


\title{
ÉLARGISSEMENT ET DÉPLACEMENT DES RAIES DE RÉSONANCE MAGNÉTIQUE CAUSÉS PAR UNE EXCITATION OPTIQUE *
}

\author{
Par J. P. BARRAT, \\ Laboratoire d'Optique, Faculté des Sciences, Caen.
}

et C. COHEN-TANNOUDJI,

Laboratoire de Physique de l'E. N. S., 24 , rue Lhomond, Paris (5e).

\begin{abstract}
Résumé. - L'effet global des processus d'absorption et d'émission de photons de résonance optique sur l'état fondamental d'un ensemble d'atomes est étudié à partir d'équations d'évoluiion établies précédemment. On met ces équations sous une forme plus simple grâce à une " approximation séculaire ", toujours valable dans les cas pratiques. On tient compte également de l'effet éventuel d'un champ de radiofréquence. Certains résultats sont établis, tels que : la description du pompage optique comme un processus de relaxation dans l'état fondamental ; la possibilité d'une conservation partielle de la cohérence au cours du cycle de pompage ; la prévision de certains déplacements des raies de résonance magnétique sous l'influence de l'excitation optique ; l'expression des signaux de détection optique à partir des éléments de la matrice densité dans l'état fondamental.
\end{abstract}

Abstract. - The total effect of absorption and emission of resonance radiation on the ground state of an ensemble of atoms is derived from equations established in an earlier paper. These equations are put in a simpler form by means of a " secular approximation" which is satisfied in all practical cases. The effect of a radiofrequency field is taken into account. Some of the results obtained are : description of optical pumping as a relaxation process in the ground state ; possibility of a partial conservation of coherence during the pumping cycle ; prediction of some shifts of the magnetic resonance lines under the influence of the optical excitation; expression for the optically detected signals as a function of the elements of the density matrix in the ground state.

\section{IV. ÉVOLUTION GLOBALE DE L'ETAT FONDAMENTAL.}

A. Équations d'évolution. - 1. Établissement DES ÉQUATIONS. - Pour obtenir l'évolution globale de la matrice densité $p$ représentant l'ensemble des atomes dans l'état fondamental, nous ajoutons les effets de l'excitation optique, décrits par (II, 13), et ceux de la retombée, décrits par (III, 10), obtenant:

$$
\begin{aligned}
& \frac{\mathrm{d}}{\mathrm{d} t} \rho_{\mu \mu^{\prime}}=-\left(\frac{1}{2 T_{p}}+i \Delta E^{\prime}\right) \sum_{\mu^{\prime \prime}} A_{\mu \mu^{\prime \prime}} f \mu^{\prime \prime} \mu^{\prime} \mathrm{e}^{i\left(\mu-\mu^{\prime \prime}\right) \omega_{\mathrm{f}} t} \\
& \quad-\left(\frac{1}{2 T_{p}}-i \Delta E^{\prime}\right) \sum_{\mu^{\prime \prime}} A_{\mu^{\prime \prime} \mu^{\prime}} \rho_{\mu \mu^{\prime \prime}} \mathrm{e}^{i\left(\mu^{\prime \prime}-\mu^{\prime}\right) \omega_{\mathrm{f}} t} \\
& \quad+\frac{\Gamma}{T_{p}} \sum_{\mu^{\prime \prime} \mu^{\prime \prime \prime}} \rho_{\mu^{\prime \prime} \mu^{\prime \prime \prime}} \\
& \left.\mathrm{e}^{i\left[\left(\mu-\mu^{\prime}\right)-\left(\mu^{\prime \prime}-\mu^{\prime \prime \prime}\right)\right] \omega_{\mathrm{f}} t} \frac{B_{\mu^{\prime \prime} \mu^{\prime \prime \prime}}^{\mu \mu^{\prime}}}{\Gamma+i\left[\left(\mu-\mu^{\prime}\right) \omega_{\mathrm{e}}-\left(\mu^{\prime \prime}-\mu^{\prime \prime \prime}\right) \omega_{\mathrm{f}}\right.}\right]
\end{aligned}
$$

Le système d'équations différentielles linéaires auquel obéissent les $\rho_{\mu \mu^{\prime}}$ a pour coefficients soit des constantes d'ordre de grandeur $\frac{1}{T_{p}}$ ou $\Delta E^{\prime}$, soit des

$\left({ }^{*}\right)$ Cette étude fait suite à un premier article paru dans le précédent numéro du Journal de Physique [1]. constantes de même ordre de grandeur multipliées par des exponentielles $\mathrm{e}^{i r \omega_{\mathrm{f}} t}(r$ entier).

2. 'Résolution' des ÉQuations (IV-1) Par approximations sugcessives. Approximation séGUlaIRE. - Nous montrons en appendice I que le système obtenu (IV-2) en ne-gardant que les coefficients constants du second membre des équations (IV-1),

$$
\begin{aligned}
& \frac{\mathrm{d}}{\mathrm{d} t} \rho_{\mu \mu^{\prime}}=-\left[\left(\frac{1}{2 T_{p}}+i \Delta E^{\prime}\right) A_{\mu \mu}\right. \\
& \left.+\left(\frac{1}{2 T_{p}}-i \Delta E^{\prime}\right) A_{\mu^{\prime} \mu^{\prime}}\right] \rho_{\mu \mu^{\prime}} \\
& +\frac{\Gamma}{T_{p}} \sum_{\substack{\mu^{\prime \prime} \mu^{\prime \prime \prime} \\
\mu^{\prime \prime}-\mu^{\prime \prime \prime}=\mu-\mu^{\prime}}} \frac{b_{\mu^{\prime \prime} \mu^{\prime \prime \prime}}^{\mu \mu^{\prime}}}{\Gamma+i\left(\mu-\mu^{\prime}\right)\left(\omega_{\mathrm{e}}-\omega_{\mathrm{f}}\right)} \rho_{\mu^{\prime \prime} \mu^{\prime \prime \prime}}
\end{aligned}
$$

a pour solution une solution approchée de (IV-1), lorsque les condition (IV-3)

$$
\left\{\begin{array}{l}
\omega_{\mathrm{f}} \mid \Delta E^{\prime} \gg 1 \\
\omega_{\mathrm{f}} T_{p} \gg 1
\end{array}\right.
$$

sont satisfaites. L'erreur commise sur la solution si l'on remplace (IV-1) par (IV-2) est de l'ordre de $1 / \omega_{\mathrm{f}} T_{p}$. Cette approximation, dite " approximation séculaire ", sera faite dans tout ce qui suit. Physiquement, les termes exponentiels $e^{i r \omega_{f} t}$ oscillent trop rapidement (à la fréquence de Larmor 
$\omega_{\mathrm{f}} / 2 \pi$ de l'état fondamental) pour qu'ils puissent avoir un effet appréciable sur l'évolution de $p$ qui n'est notable que pour des temps de l'ordre de $1 / \Delta E^{\prime}, T_{p} \gg 2 \pi / \omega_{\mathrm{f}}$.

3. Justification DES APPROXimations, Faites. - Nous venons de voir que $\rho_{\mu \mu^{\prime}}$ varie avec une vitesse caractérisée par les temps $T_{p}$ et $1 / \Delta E^{\prime}$.

Or nous avons supposé dans le calcul de l'expression (II-4) que $\rho_{\mu \mu^{\prime}}$ variait très lentement sur des intervalles de temps de l'ordre de $1 / \Delta$. Cette hypothèse est justifiée puisque d'après (I-1) et (IV-3)

$$
1 / \Delta \ll 1 / \omega_{\mathfrak{f}} \ll T_{p}, \quad 1 / \Delta E^{\prime} .
$$

D'autre part, dans le calcul de l'expression (III, 10) nous avons admis que $p_{\mu \mu^{\prime}}$ variait lentement devant la durée de vie $\tau \stackrel{=}{=} 1 / \Gamma$ de l'état excité. Nous devons donc compléter les conditions de validité (IV-3) par

$$
\left\{\begin{array}{l}
\Gamma \mid \Delta E^{\prime} \gg 1 \\
\Gamma T_{p} \gg 1
\end{array}\right.
$$

Les conditions (IV-3) et (IV-4) expriment physiquement que le déplacement $\Delta E^{\prime}$ et la largeur $1 / T_{p}$ d'origine optique des raies de résonance magnétique sont petits devant la fréquence $\omega_{\mathrm{f}} / 2 \pi$ de ces raies (fréquence de Larmor de l'état fondamental), et devant la largeur naturelle du niveau excité. Elles portent en fait sur la fréquence $\omega_{\mathrm{f}} / 2 \pi$ et sur le flux lumineux de pompage. Elles sont toujours remplies dans les expériences usuelles.

Il faut y ajouter la condition (I-1) $\Delta \gg \omega_{\mathrm{e}}, \omega_{\mathrm{f}}$, $\Gamma$. On pourrait s'affranchir de la restriction $\Delta \gg \omega_{\mathrm{e}}$, $\omega_{\mathrm{f}}$ en compliquant l'écriture des équations.

4. Matrice Densité Dans L'ÉtÁt EXcité. Les approximations (IV-3), qui impliquent aussi pratiquement $\omega_{\mathrm{e}} T_{p} \gg 1$ permettent d'intégrer (II-18), en cherchant directement la solution forcée de l'équation avec second membre et en négligeant la variation de $\rho_{\mu \mu^{\prime}}$ devant l'exponentielle

On trouve

$$
\mathrm{e}^{i\left[\left(\left[m-m^{\prime}\right) \omega_{\mathrm{e}}-\left(\mu-\mu^{\prime}\right) \omega_{\mathrm{f}}\right] t\right.}
$$

$$
\begin{aligned}
f_{m m^{\prime}}(t)=\frac{1}{T_{p}} \sum_{\mu \mu^{\prime}} \rho_{\mu \mu^{\prime}}(t) \mathrm{e}^{\left.i l\left(m-m^{\prime}\right) \omega_{\mathrm{e}}-\left(\mu-\mu^{\prime}\right) \omega_{\mathrm{f}}\right] t} & \\
& \frac{\left\langle m\left|\mathbf{e}_{\mathrm{\lambda}} D\right| \mu><\mu^{\prime}\left|\mathbf{e}_{\lambda_{0}} . D\right| m^{\prime}>\right.}{\Gamma+i\left[\left(m-m^{\prime}\right) \omega_{\mathrm{e}}-\left(\mu-\mu^{\prime}\right) \omega_{\mathfrak{f}}\right]} .
\end{aligned}
$$

Ce résultat, joint à (III-4), permet de calculer les intensités de la lumière de fluorescence réémises dans les différentes directions. Nous avons également vu (§ II) comment calculer la quantité de lumière absorbée. Notre formalisme permet donc de décrire l'évolution de l'état fondamental sous l'influence du pompage optique, et de calculer d'autre part les signaux de détection optique.

B. Interprétation physique. - 1 . Structure DES Équations. - Les équations (IV-2) montrent qu'un élément $\rho_{\mu \mu^{\prime}}$ de la matrice densité est couplé à tous les autres éléments $P_{\mu^{\prime \prime}} \mu^{\prime \prime \prime}$ tels que

$$
\mu-\mu^{\prime}=\mu^{\prime \prime}-\mu^{\prime \prime \prime} \text {. }
$$

Elles se divisent donc en groupes distincts, $2 I+1$ équations couplées pour $\mu-\mu^{\prime}=0$, $2 I$ pour $\mu-\mu^{\prime}=1$, et ainsi de suite. Elles sont, à cet égard, très analogues aux équations de relaxation de Bloch-Ayant-Kubo-Tomita [2] décrivant l'évolution d'un ensemble de spins soumis à une interaction aléatoire avec le réseau, qui varie assez vite pour que l'approximation de rétrécissement par le mouvement soit valable. L'approximation séculaire est également utilisée dans ce cas, la condition $\omega_{\mathrm{f}} T_{p} \gg 1$ s'écrit alors $\omega_{\mathrm{f}} T \gg 1$ où $T$ est le temps de relaxation.

2. Évolution des populations. - Les populations des niveaux $\mu$ sont les $p_{\mu \mu}$, qui satisfont à

$$
\frac{\mathrm{d} \rho_{\mu \mu}}{\mathrm{d} t}=-\frac{A_{\mu \mu}}{T_{p}} \rho_{\mu \mu}+\frac{1}{T_{p}} \sum_{\mu^{\prime}} B_{\mu^{\prime} \mu^{\prime}}^{\mu \rho^{\prime}} \rho_{\mu^{\prime} \mu^{\prime}} .
$$

On pose

$$
\begin{aligned}
P_{\mu^{\prime}} \rightarrow \mu & =\frac{B_{\mu^{\prime} \mu^{\prime}}^{\mu \mu}}{T_{p}}=\frac{1}{T_{p}} \sum_{m} \mid
\end{aligned}
$$

$\frac{1}{T_{p}^{\prime}}\left|<\mu^{\prime}\right| e_{\lambda_{0}} \cdot D|m>|^{2}$ est la probabilité par unité de-temps de passage du niveau $\mu^{\prime}$ au niveau $m$ par absorption d'un photon ;

$$
\left|C_{1 I}(F, m ; m-\mu, \mu)\right|^{2}
$$

est la probabilité relative de passage d'un niveau $m$ déterminé à l'un des niveaux $\mu$ par émission spontanée. $P_{\mu^{\prime} \rightarrow \mu}$ représente donc la probabilité par unité de temps d'un passage du niveau $\mu^{\prime}$ au niveau $\mu$ par résonance optique. D'ailleurs, on sait que $\sum_{\mu}\left|C_{1 I}(F, m ; m-\mu, \mu)\right|^{2}=1$ et l'on peut écrire :

$$
\begin{aligned}
\frac{A_{\mu \mu}}{T_{p}} & =\frac{1}{T_{p}} \sum_{m}|<m| \mathbf{e}_{\lambda_{0}} . D|\mu>|^{2} \\
& =\frac{1}{T_{p}} \sum_{m \mu^{\prime}}|<m| e_{\lambda_{0}} . D|\mu>|^{2}\left|C_{1 I}\left(F, m ; m-\mu^{\prime}, \mu^{\prime}\right)\right|^{2} \\
& =\sum_{\mu^{\prime}} P_{\mu \rightarrow \mu^{\prime}} \quad \text { (IV-8 }
\end{aligned}
$$

(IV-6) s'écrit alors :

$$
\frac{\mathrm{d} \rho_{\mu \mu}}{\mathrm{d} t}=-\left(\sum_{\mu^{\prime}} P_{\mu \rightarrow \mu^{\prime}}\right) \rho_{\mu \mu}+\sum_{\mu^{\prime}}\left(P_{\mu^{\prime}} \rightarrow \mu\right) \rho_{\mu^{\prime} \mu^{\prime}} \quad(\mathrm{IV}-9)
$$

L'interprétation en est alors évidente : la variation de la population $\rho_{\mu \mu}$ du niveau $\mu$ est égale, par unité de temps, à tout ce qui arrive des autres niveaux $\mu^{\prime}$, diminué de tout ce qui part vers les autres niveaux $\mu^{\prime}$. Ceci est à rapprocher des équa- 
tions obtenues en théorie de la relaxation. La principale différence est que, dans notre cas,

$$
P_{\mu \rightarrow \mu^{\prime}} \neq P_{\mu^{\prime} \rightarrow \mu}
$$

au moins en général. Si bien que l'on peut obtenir des différences de populations appréciables entre les niveaux par suite de l'absorption et de la reémission de photons. C'est le principe même du pompage optique. La matrice des $(2 I+1)$ quantités $P_{\mu \rightarrow \mu^{\prime}}$ n'est autre que la matrice de pompage optique déjà utilisée par divers auteurs [3].

Remarquons que bien entendu

$$
\frac{\mathrm{d}}{\mathrm{d} t} \text { Trace } \rho=\frac{\mathrm{d}}{\mathrm{d} t} \sum_{\mu} \rho_{\mu \mu}=0,
$$

c'est-à-dire que la somme des populations de l'état fondamental est constante.

3. Évolution de la cohérence. Cas partiCULIER DE 2 Niveaux. - Dans ce cas, qui est par exemple celui de l'isotope ${ }^{199} \mathrm{Hg}$, la matrice $\rho$ ne comporte qu'un seul élément non diagonal, $\rho_{\frac{1}{2}}-\frac{1}{2}$ (que nous ne distinguons pas de $p_{-\frac{1}{2} \frac{1}{2}}$ qui en est complexe conjugué), puisqu'il n'y a que 2 sousniveaux Zeeman $\left(I=\frac{1}{2}, \mu= \pm \frac{1}{2}\right)$. L'équation (IV-2) s'écrit alors :

$$
\begin{aligned}
\frac{\mathrm{d}}{\mathrm{d} t} \rho_{\frac{1}{2}-\frac{1}{2}}=-f_{\frac{1}{2}-\frac{1}{2}}\left[\frac{\left[\begin{array}{l}
A_{\frac{1}{2}}+A \\
T_{p}
\end{array}\right.}{+i \Delta E^{\prime}\left(A_{\frac{1}{2} \frac{1}{2}}-A_{-\frac{1}{2}-\frac{1}{2}}\right)}\right] \\
+\frac{\Gamma}{T_{p}} \frac{\frac{1}{2}-\frac{1}{2}}{\Gamma+i\left(\omega_{\mathrm{e}}-\omega_{\mathrm{f}}\right)} \rho_{\frac{1}{2}-\frac{1}{2}} \cdot \quad(\mathrm{IV}-10)
\end{aligned}
$$

D'où :

$$
\rho_{\frac{1}{2}-\frac{1}{2}}(t)=\rho_{\frac{1}{2}-\frac{1}{2}}\left(t_{0}\right) \mathrm{e}^{-\left(\frac{1}{T_{2}}+i \varepsilon\right)\left(t-t_{0}\right)}
$$

avec

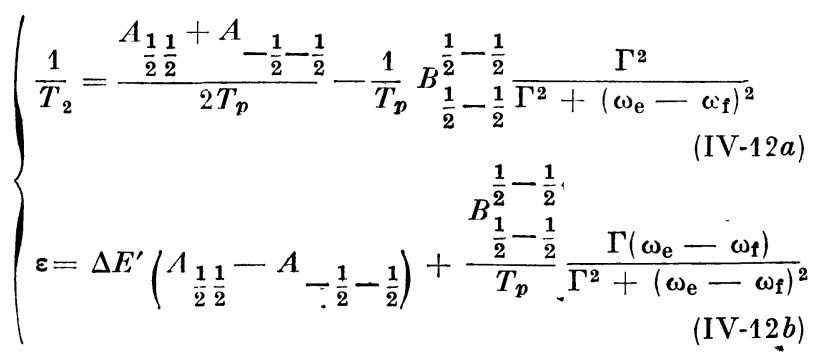

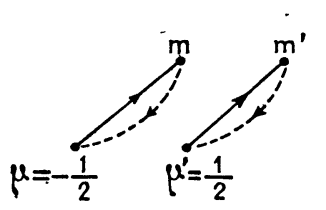

Fig. $1 a$.

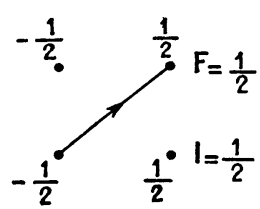

Fig, $1 b$, a) Interprétation de $T_{2}: T_{2}$ est la constante de temps avec laquelle le module de $\rho_{\frac{1}{2}-\frac{1}{2}}$ décroît. $1 / T_{2}$ est la probabilité par unité de temps de disparition de la cohérence. $1 / T_{2}$ comprend 2 termes. Le $1^{\mathrm{er}}$,

$$
\frac{1}{T_{2}^{\prime}}=\frac{A_{\frac{11}{2}}{ }^{-} A_{-\frac{1}{2}-\frac{1}{2}}}{2 T_{p}}
$$

est la demi-somme des inverses des durées de vie $1 / T_{\frac{1}{2}}=A_{\frac{11}{2}} / T_{p}, 1 / T_{-\frac{1}{2}}=A_{-\frac{1}{2}-\frac{1}{2}} / T_{p}$ des niveaux $\mu={ }^{2}+1 / 2$ et $\mu=-1 / 2$. Il représente la disparition de la cohérence sous l'effet de l'excitation optique qui arrache les atomes à la superposition cohérente dans laquelle ils se trouvent. On peut dire qu'il représente la contribution de la relaxation longitudinale à la relaxation transversale.

Le $2^{\mathrm{e}}$ terme :

$$
-\frac{1}{T_{2}^{\prime \prime}}=-\frac{B_{\frac{1}{2}-\frac{1}{2}}^{\frac{1}{2}-\frac{1}{2}}}{T_{p}} \frac{\Gamma^{2}}{\Gamma^{2}+\left(\omega_{\mathrm{e}}-\omega_{\mathrm{p}}\right)^{2}}
$$

représente la fraction de la cohérence qui s'est conservée au cours du pompage et qui se retrouve, après absorption et émission d'un photon, dans l'état fondamental. Nous montrons dans l'appendice II qu'il est toujours négatif, et inférieur en module au $1^{\text {er }}$ terme. D'après l'expression (III-11) de $B_{\mu^{\prime \prime} \mu^{\prime \prime \prime \prime}}^{\mu \mu^{\prime}}$, cet effet n'existe que s'il existe 2 transitions de même nature $\left(\pi, \sigma_{+}\right.$ou $\left.\sigma_{-}\right)$, donc parallèles dans le diagramme de Grotrian partant respectivement des niveaux $1 / 2$ et $-1 / 2$ (fig. $1 a)$. Ainsi il n'existe pas pour la composante hyperfine de la raie $2537 \AA$ partant du niveau $F=1 / 2 \mathrm{du}^{199} \mathrm{Hg}$ en excitation $\sigma_{+}(f i g .1 b)$, mais il existe pour la composantehyperfine partant du niveau $F=3 / 2$ (fig. 1c). La variation de cet effet avec le champ magnétique suit une loi analogue à celle de l'effet Hanle [4]. On doit pouvoir l'observer expérimentalement en constatant une diminution de la contribution de l'intensité lumineuse à la largeur des raies de résonance magnétique lorsqu'on diminue le champ magnétique.

b) Interprétation de $\varepsilon$ : La matrice densité $\sigma(t)$ en représentation de $\mathrm{Sch}^{0}{ }^{0}$ dinger est liée à $\rho(t)$, matrice densité en représentation d'interaction, par

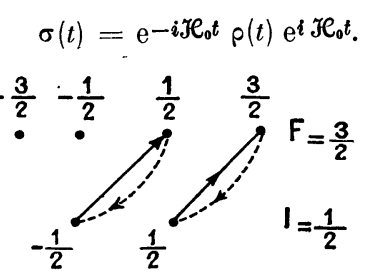

Fị. $1 c$, 
Done

$$
\sigma_{\mu, \mu^{\prime}}(t)=p_{\mu \mu^{\prime}}(t) \mathrm{e}^{-i\left(E_{\mu}-E_{\mu, \mu)}\right.}
$$

et dans le cas examiné :

$$
\sigma_{\frac{1}{2}-\frac{1}{2}}(t)=\sigma_{\frac{1}{2}-\frac{1}{2}}\left(t_{0}\right) \mathrm{e}^{-\left(\frac{1}{T_{\mathrm{s}}}+i \omega_{\mathrm{f}}+i \varepsilon\right)\left(t-t_{0}\right)}
$$

$\varepsilon$ représente donc un changement de la différence d'énergie entre les sous-niveaux Zeeman. D'après '(IV-12b), $\varepsilon$ comprend aussi 2 termes.

Le $1^{\text {er }}$ terme $\Delta E^{\prime}\left(A_{\frac{1}{2} \frac{1}{2}}-A_{-\frac{1}{2}-\frac{1}{2}}\right)$ représente le déplacement dû à la différence des selfs-énergies $\Delta E^{\prime} A_{\frac{1}{2} \frac{1}{2}}$ et $\Delta E^{\prime} A_{-\frac{1}{2}-\frac{1}{2}}$ des 2 sous-niveaux $\pm 1 / 2 *$. Nous avons déjà donné plus haut l'interprétation physique de $\Delta E^{\prime}(\S$ II, 4 ).

Remarquons que notre calcul s'applique à une composante hyperfine particulière de la raie de résonance. Dans le cas de l'isotope ${ }^{199} \mathrm{Hg}$, il s'agit par exemple de la transition $\tilde{k}_{01}$ (ou $\tilde{k}_{02}$ ) reliant le niveau hyperfin unique $F=1 / 2$ du niveau fondamental $6^{1} S_{0}$ au niveau hyperfin $F=1 / 2$ (ou $F=3 / 2)$ du niveau excité $6^{3} P_{1}$. Nous supposons le centre $k_{1}$ de la raie excitatrice suffisamment proch 3 de $\tilde{k}_{01}$ et $\tilde{k}_{02}$. Comme il s'agit d'une self-énergie, nous devons tenir compte également des transitions virtuelles vers l'autre niveau hyperfin $F=3 / 2$ (ou $F=1 / 2$ ) du niveau de résonance et vers les autres niveaux excités. Considérons l'effet des transitions virtuelles vers les niveaux hyperfins $k_{0 \mathrm{~F}}^{\prime}$ d'un autre niveau excité. $k_{1}-k_{0 \mathrm{~F}}^{\prime}$ varie alors infiniment peu d'une composante hyperfine à l'autre. D'après (II, $8, b$ ) il en est donc de' même pour $\Delta E^{\prime}$. II est facile alors de voir que par suite des règles de somme sur les $A_{\mu \mu}$, l'effet de ces transitions virtuelles est de déplacer les 2 sousniveaux $\pm 1 / 2$ de la même quantité.

Le déplacement de la raie de résonance magnétique est donc sensible uniquement aux transitions virtuelles vers l'ensemble des niveaux hyperfins du niveau excité prochę de $k_{1}$.

Le $2^{\mathrm{e}}$ terme représente un déplacement d'énergie fondamentalement différent du précédent Il est lié aux transitions réelles vers le niveau de résonance. 'Il n'existe que si une fraction de cohérence se conserve au cours du pompage Lors du temps $\tau=1 / \Gamma$ passé en moyenne dans l'état exci ié, cette fraction de cohérence " voit " un effet Zeeman $\omega_{\dot{e}}$ et non plus $\omega_{\mathrm{f}}$. D'où un déphasage de l'ordre de $\left(\omega_{\mathrm{e}}-\omega_{\mathrm{f}}\right) / \Gamma$ qu'il faut multiplier par

$$
B_{\frac{1}{2}-\frac{1}{2}}^{\frac{1}{2}-\frac{1}{\Gamma^{2}+\left(\omega_{\mathrm{e}}-\omega_{\mathrm{f}}\right)^{2}}}
$$

(") Cet effet a été récemment mis en évidence par l'un de

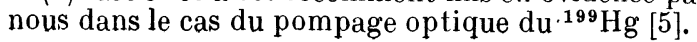

fraction de la cohérence qui se conserve $\mathrm{Si}$ le champ magnétique est grand, l'effet est petit parce qu'une faible fraction de la cohérence se conserve. S'il est petit, l'effet l'est aussi parce que les effets Zeeman et les déphasages le sont. Il y a optimum lorsque $\omega_{\mathrm{e}}-\omega_{\mathrm{f}} \sim \Gamma$. Sur le ${ }^{199} \mathrm{Hg}$, on peut imaginer des expériences où le déplacement, dû à cet effet, de la raie de résonance magnétique serait de l'ordre de $1 / 2$ de la largeur due à l'excitation optique, donc facilement observable.

4. Évolution de la cohérence dans le cas GÉNÉRAL. - Dans ce cas, on a :

$$
\begin{aligned}
& \frac{\mathrm{d}_{F \mu \mu^{\prime}}}{\mathrm{d} t}=-\left(\frac{1}{T_{2 \mu \mu^{\prime}}}+i \varepsilon_{\mu \mu^{\prime}}\right) \rho_{\mu \mu^{\prime}}
\end{aligned}
$$

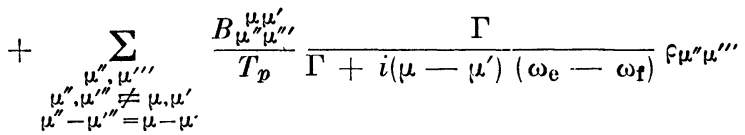

$1 / T_{2 \mu \mu^{\prime}}$ et $\varepsilon_{\mu \mu^{\prime}}$ ont des définitions analogues à (IV-12). Lour interprétation a déjà été donnée. Les termes nouveaux représentent un transfert partiel vers le couple $\left(\mu, \mu^{\prime}\right)$ de la cohérence existant dans le couple $\left(\mu^{\prime \prime}, \mu^{\prime \prime \prime}\right)$, par l'intermédiaire de l'état excité. Le système auquel satisfont les $\rho_{\mu \mu^{\prime}}$, pour $\mu-\mu^{\prime}$ fixé, est très analogue au système obtenu en théorie de la relaxation. La matrice associée à ce système [matrice d'élargissement [6]] a ses valeurs propres à partie réelle négative (cf. appendice II). La pompage fait donc disparaître la cohérence comme un processus de relaxation.

\section{EVOLUTION GLOBALE EN PRÉSENCE D'UN CHAMP DE RADIOFREQ UENCE.}

1. Position du problème. - Nous avons jusqu'à présent étudié la variation dans le temps, sous l'influence d'une excitation optique, d'un ensemble d'atomes dans l'état fondamental, représentés par une matrice densité $\rho(t)$, connaissant la matrice $\rho\left(t_{0}\right)$ non diagonale $a$ priori (c'est-à-dire qu'il peut $\mathrm{y}$ avoir cohérence à l'instant $t_{0}$ ). Nous allons maintenant étudier l'évolution de l'état fondamental sous l'action simultanée de l'excitation optique et d'un champ de radiofréquence $H_{1}$, perpendiculaire au champ statique créant l'effet Zeeman, tournant àøla fréquence $\omega$ voisine de $\omega_{\mathrm{f}}$. Nous négligerons l'action de ce champ sur l'état excité, soit que $\omega_{\mathrm{e}}$ soit très différent de $\omega_{\mathrm{f}}$, soit que la durée de vie $\tau=\Gamma^{-1}$ de l'état excité soit trop courte pour qu'il puisse subir une action sensible de la part de $H_{1}$. L'Hamiltonien représentant l'action de $H_{1}$ est :

$$
\mathfrak{K}_{R F}=\frac{\gamma H_{1}}{2}\left[I_{+} \iota-i \omega t+I_{-} \epsilon^{i \omega t}\right]
$$


où $\gamma$ est le rapport gyromagnétique de l'état fondamental, I son moment cinétique.

Indiquons dès maintenant que les équations finales que nous obtenons dans ce cas sont analogues aux équations (IV-2), les seules différences étant :

a) $\rho(t)$, matrice densité en représentation d'interaction, est remplacée par $\tilde{\rho}(t)$, matrice densité dans le référentiel $R$ tournant à la vitesse angulaire $\omega$ autour du champ statique :

$$
\tilde{\rho}(t)=\epsilon^{i\left(\omega-\omega_{\mathrm{f}}\right) I z t} \quad \rho(t) \epsilon^{-i\left(\omega-\omega_{\mathrm{f}}\right)} z_{z} t
$$

b) il apparaît au second membre un terme

$$
-i[H, \tilde{\rho}]_{\mu, \mu^{\prime}}
$$

où

$$
\mathscr{H}=\left(\omega_{\mathrm{f}}-\omega\right) I_{z}+\gamma H_{1} J_{x}
$$

$(\mathrm{V}-3)$.

représente l'effet du champ statique et $d u$ champ $H_{1}$ dans le référentiel $R$.

c) $\omega_{\mathfrak{f}}$ doit, dans les équations, être remplacé par $\omega$.

Pour établir ces résultats, nous étudierons successivement le processus d'absorption (cf. § IJ) et celui de réémission (cf. § III).

2. Equations du problème. - Au lieu de passer en représentation d'interaction pour l'état fondamental et l'état excité, nous effectuerons la transformation qui consiste à passer dans le référentiel tournant $R$ pour l'état fondamental, et en représentation d'interaction pour ce qui concerne l'état excité et le rayonnement. On pose :

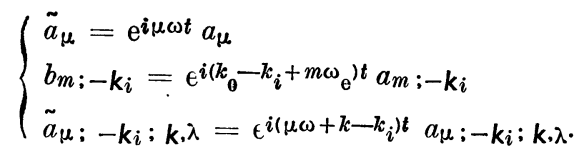

Cette transformation remplace $\mathfrak{H}_{R F}$ par $\mathfrak{H}$ et les équations de départ (I-9) deviennent, avec cet Hamiltonien supplémentaire :

$$
\begin{aligned}
& \left|i \dot{\tilde{a}}_{\mu}=\sum_{\mu^{\prime}} \mathcal{X}_{\mu \mu^{\prime}} \tilde{a}_{\mu^{\prime}}+\sum_{m, i}<\mu, k_{i}, \lambda_{0}\right| \tilde{\mathfrak{H}}_{I} \mid m>b_{m} ;-k_{i} \\
& i b_{m:-k_{i}}=\sum_{\mu^{\prime}}<m\left|\tilde{\mathscr{H}}_{I}\right| \mu^{\prime}, k_{i}, \lambda_{0}>\tilde{a}_{\mu^{\prime}}
\end{aligned}
$$

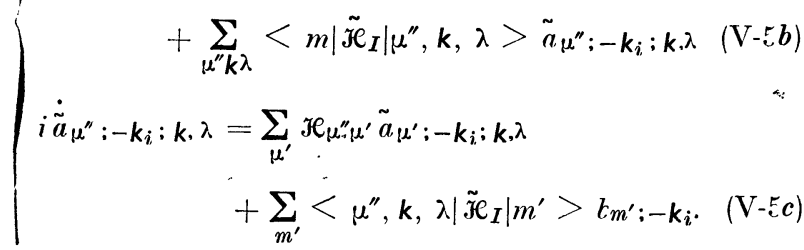

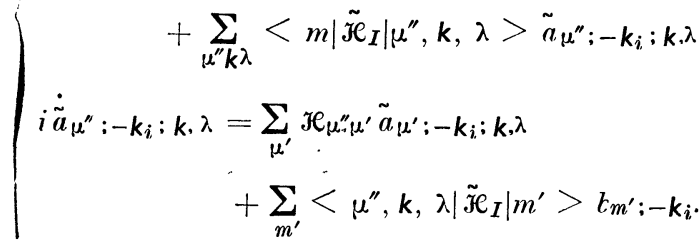

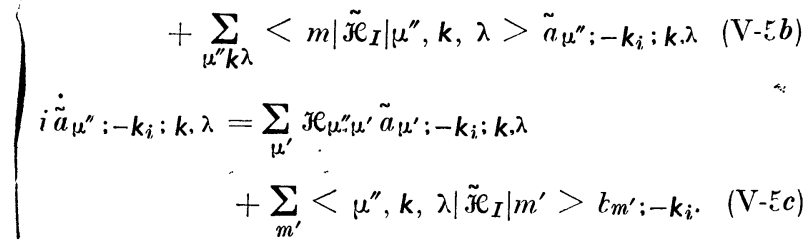

Les élém€nts de matrice de $\tilde{\mathscr{H}}_{I}$ sont obtenus $€$ n remplaçant $\omega_{\mathrm{f}}$ par $\omega$ dans les expressions (I-10) de ceux de $\mathfrak{H}_{I}^{\prime}$. On a posé $\mathfrak{H}_{\mu \mu^{\prime}}=\left\langle\mu|\mathfrak{H}| \mu^{\prime}\right\rangle$.
3. Processus d'absorption. - Comme au " II, on intègre $(\mathrm{V}-5 c)$ et on report ‘ dans $(\mathrm{V}-5 b)$ obtenant

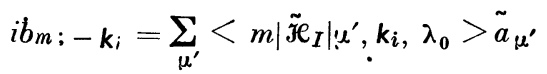

$$
\begin{aligned}
& -i \sum_{\substack{\mu^{\prime \prime} k \lambda \\
\mu m^{\prime}}} \int_{t_{\mathrm{o}}}^{t} \mathrm{~d} t^{\prime}\left\langle m\left|\tilde{\mathscr{H}}_{I}(t)\right| \mu^{\mu \prime}, k, \lambda>\right. \\
& <\mu^{\prime \prime}\left|\mathrm{e}-i \mathscr{H}\left(t-t^{\prime}\right)\right| \mu><\mu, k, \lambda\left|\tilde{\mathscr{H}}_{I}\left(t^{\prime}\right)\right| m^{\prime}>b_{m^{\prime}} ;-k_{i}\left(t^{\prime}\right) .
\end{aligned}
$$

Le calcul s'effectue alors de la manière classique indiquée au § II. Si l'on explicitè les éléments de matrice de $\tilde{H}_{I}$, on voit apparaître une exponentielle $\mathrm{e}^{i\left[k_{0}-k+m^{\prime} \omega_{\mathrm{e}}-\mu \omega-\Im \mathcal{K}\right]\left(t-t^{\prime}\right)} ;$ comme $k_{0}$ est très supérieur aux éléments de matrice de $\mathscr{H}$, ̀̀ $m^{\prime \prime} \omega_{\mathrm{e}}$ et à $\mu \omega$, on peut remplacer cette exponentielle par $e^{i\left(k_{0}-k\right)\left(t-t^{\prime}\right)}$ ce qui revient à remplacer $<\mu^{i \prime \prime}\left|e^{-i \mathscr{H}\left(t-t^{\prime}\right)}\right| \mu>$ par $\left\langle\mu^{\prime \prime} \mid \mu\right\rangle=\delta_{\mu^{\prime \prime} \mu}$. Il reste alors

$$
-i\left(\frac{\Gamma}{2}+i \Delta E\right) b_{m:-k_{i}}(t) .
$$

Ce résultat était prévisible : la radiofréquence agissant sur l'état fondamental ne modifie ni la durée de vie, ni la self-énergie de l'état excité. On a done :

$$
\begin{aligned}
i b_{m}:-k_{i}= & -i\left(\frac{\Gamma}{2}+i \Delta E\right) b_{m:-k_{i}} \\
& +\sum_{\mu^{\prime}}<\dot{m}\left|\tilde{\mathscr{H}}_{I}\right| \mu^{\prime} k_{i} \lambda_{0}>\tilde{a}_{\mu^{\prime}}
\end{aligned}
$$

L'élimination de $b_{m:-k_{i}}$; entre (V-7) et (V-5a) se fait alors de manière identique à ce qui a été fait au $\S$ II, puisque $\mathscr{H}$ n'intervient pas. Elle conduit à

$$
\begin{aligned}
& \frac{\mathrm{d}^{(1)} \mathrm{d} t}{\mathrm{\rho}}{\tilde{\mu \mu^{\prime}}}=-i[\mathcal{H}, \tilde{\rho}]_{\mu \mu^{\prime}} \\
& \quad-\left(\frac{1}{\sqrt{T_{p}}}+i \Delta E^{\prime}\right) \sum_{\mu^{\prime \prime}} A_{\mu \mu \mu^{\prime \prime}} \tilde{\rho}_{\mu^{\prime \prime} \mu^{\prime} c^{\prime}} i\left(\mu-\mu^{\prime \prime}\right) \omega t \\
& \quad-\left(\frac{1}{2 T_{p}}-i \Delta E^{\prime}\right) \sum_{\mu^{\prime \prime}} A_{\mu^{\prime \prime} \mu^{\prime}} \tilde{\rho}_{\mu \mu^{\prime \prime}} \mathrm{e}^{i\left(\mu^{\prime \prime}-\mu^{\prime}\right) \omega t .}
\end{aligned}
$$

On trouvera de même

$$
\begin{aligned}
\frac{\mathrm{d}}{\mathrm{d} t} F_{m m^{\prime}}=- & \Gamma_{F m m^{\prime}}+\frac{1}{T_{\boldsymbol{p}}} \sum_{\mu \mu^{\prime}} \epsilon^{\left.i\left[m-m^{\prime}\right) \omega_{\mathrm{e}}-\left(\mu-\mu^{\prime}\right) \omega\right] t} \\
& <m\left|\mathbf{e}_{\lambda_{0}} D\right| \mu><\mu^{\prime}\left|\mathbf{e}_{\lambda_{0}} D\right| m^{\prime}>_{f \mu \mu^{\prime}} .
\end{aligned}
$$

4. Processus de réémission. - On généralise facilement le calcul du $\S$ (III, 1). On intègrè (V-5c), on prend le complexe conjugué de l'expression obtenue et on le multiplie membre à membre par $(\mathrm{V}-5 c)$. On retrouve les mêmes simplifications que dans le calcul fait ci-dessus au $\S$ III, en raison du fait que l'émission spontanée ne dépend pas de la radio- 
fréquence dans l'état fondamental. On obtient ainsi :

$$
\begin{aligned}
& \frac{\mathrm{d}^{(2)} \tilde{\rho}_{\mu \mu^{\prime}}}{\mathrm{d} t}=-i[\mathcal{H}, \tilde{\rho}]_{\mu, \mu^{\prime}} \\
& +\sum_{\substack{m m^{\prime} \\
m-m^{\prime}=\mu-\mu^{\prime}}} \mathrm{e}^{i\left(\mu^{\prime}-\mu\right)\left(\omega_{\mathrm{e}}-\omega\right) t} C_{1 I}(F, m ; m-\mu, \mu) \\
& \quad C_{1 I}\left(F, m^{\prime}, m^{\prime}-\mu^{\prime}, \mu^{\prime}\right) \rho_{m m^{\prime}}(t) .
\end{aligned}
$$

L'élimination de $\rho_{m m^{\prime}}$ entre (V-9) et (V-10) donne alors

$$
\begin{aligned}
& \frac{\mathrm{d}^{(2)} \tilde{\rho}_{\mu \mu^{\prime}}}{\mathrm{d} t}=-i[\tilde{r}, \tilde{\rho}]_{\mu \mu^{\prime}} \\
& \quad+\frac{\Gamma}{T_{p}} \sum_{\mu^{\prime \prime} \mu^{\prime \prime \prime}} \frac{\tilde{\rho} \mu_{\mu^{\prime \prime} \mu^{\prime \prime \prime}} \mathrm{e}^{i\left[\left(\mu-\mu^{\prime}\right)-\left(\mu^{\prime \prime}-\mu^{\prime \prime \prime}\right)\right] \omega t} \bar{b}_{\mu^{\prime \prime} \mu^{\prime \prime \prime}}^{\mu \mu^{\prime}}}{\Gamma+i\left[\left(\mu-\mu^{\prime}\right) \omega_{\mathrm{e}}-\left(\mu^{\prime \prime}-\mu^{\prime \prime \prime}\right) \omega\right]} .
\end{aligned}
$$

5. Equations d'évolution complètes. - En ajoutant (V-8) et (V-11) (en ne gardant bien entendu qu'une fois le terme contenant Je), on obtient l'équation d'évolution globale de $\tilde{\rho}$. On arrive aux équations (V-12)

$$
\begin{aligned}
& \frac{\mathrm{d} \tilde{\rho}_{\mu \mu^{\prime}}}{\mathrm{d} t}=-i[\mathrm{He}, \tilde{\rho}]_{\mu \mu^{\prime}} \\
& -\left[\frac{1}{2 T_{p}}\left(A_{\mu \mu}+A_{\mu^{\prime} \mu^{\prime}}\right)+i \Delta E^{\prime}\left(A_{\mu \mu}-A_{\mu^{\prime} \mu^{\prime}}\right)\right] \tilde{\rho}_{\mu \mu^{\prime}} \\
& +\frac{1}{T_{p}} \sum_{\substack{\mu^{\prime \prime} \mu^{\prime \prime \prime} \\
\mu^{\prime \prime}-\mu^{\prime \prime \prime}=\mu-\mu^{\prime}}} \frac{\Gamma}{\Gamma+i\left(\mu-\mu^{\prime}\right)\left(\omega_{\mathrm{e}}-\omega\right)} b_{\mu^{\prime} \mu^{\prime \prime \prime}}^{\mu \mu^{\prime}} \tilde{\rho}_{\mu^{\prime \prime} \mu^{\prime \prime \prime}}
\end{aligned}
$$

en utilisant toujours l'approximation séculaire qui impose la condition de validité supplémentaire $(\mathrm{V}-13): \gamma H_{1} \ll \omega$. (Les éléments de matrice de $\mathfrak{H}$ étant de l'ordre de $\gamma H_{1}$.)

Cette condition exprime que la largeur due à la radiofréquence des raies de résonance magnétique est petite devant la fréquence de ces raies.

Le système (V-12) montre bien que le pompage optique peut être globalement considéré comme un processus de relaxation. Il montre que les termes discutés en (IV-3b) interviennent bien pour déplacer les raies de résonance magnétique. Il devrait être complété par l'introduction (plus ou moins phénoménologique) de termes décrivant la relaxation proprement dite (désorientation des atomes par collision contre les parois, les molécules d'un gaz étranger, etc...). La résolution du système (V-12) permet de calculer les signaux de détection optique.

6. Signaux de détection optique. - a) Lumière absorbée par unité de temps ; elle s'obtient aisément en prenant la trace de (V-8)

$$
\begin{aligned}
& L_{A}=\sum_{\mu}-\frac{\mathrm{d}^{(1)}}{\mathrm{d} t} \tilde{\rho}_{\mu \mu} \\
& =+\left(\frac{1}{2 T_{p}}+i \Delta E^{\prime}\right) \cdot \sum_{\mu^{\prime \prime \mu}} A_{\mu \mu \mu^{\prime \prime}} \tilde{\rho}_{\mu^{\prime \prime} \mu} \mathrm{e}^{i\left(\mu-\mu^{\prime \prime}\right) \omega t}+\quad+\text { complexe conjugué, }
\end{aligned}
$$

On voit qu'une modulation peut apparaitre aux fréquences $r \omega / 2 \pi$ ( $r$ entier) (expériences de faisceau croisé [7]).

b) Lumière de fluorescence émise par unité de temps, dans une direction et avec un état de polarisation donné. On l'obtient en calculant

$$
L_{F}=\frac{\mathrm{d}}{\mathrm{d} t} \sum_{\mu i|k|}\left[\tilde{a}_{\mu ;-k_{i} ; k, \lambda} \tilde{a}_{\mu ;-k_{i} ; k, \lambda}\right]
$$

$=\frac{3}{8 \pi} \Gamma \sum_{\mu m m^{\prime}} \mathrm{e}^{i\left(m^{\prime}-m\right) \omega e t<\mu\left|\mathbf{e}_{\lambda} . D\right| m}>_{f_{m m^{\prime}}}<m^{\prime}\left|\mathbf{e}_{\lambda} D\right| \boldsymbol{\mu}>$

avec $\rho_{m m^{\prime}}$ donné par la généralisation de (IV-5)

$$
\begin{aligned}
\rho_{m m^{\prime}}=\frac{1}{T_{p}} \sum_{\mu \mu^{\prime}} \tilde{f}_{\mu \mu^{\prime}}(t) \mathrm{e}^{i\left[\left(m-m^{\prime}\right) \omega_{\mathrm{e}}-\left(\mu-\mu^{\prime}\right) \omega\right] t} & <\dot{m}\left|\mathbf{e}_{\lambda_{0}} D\right| \mu><\mu^{\prime}\left|\mathbf{e}_{0} \cdot D\right| m^{\prime}> \\
& \frac{}{\Gamma+i\left[\left(m-m^{\prime}\right) \omega_{\mathrm{e}}-\left(\mu-\mu^{\prime}\right) \omega\right]} .
\end{aligned}
$$

\section{CONCLUSION}

En résumé, nous pouvons classer les résultats de cette étude en 3 parties :

1. Vue de l'état fondamental, et en présence ou non de radiofréquence, l'excitation optique par la raie de résonance peut être décrite mathématiquement et physiquement comme un processus de relaxation qui modifie la répartition des populations et détruit la cohérence. Cette destruction n'est cependant pas totale au cours d'un cycle de pompage: Une fraction de la cohérence peut se conserver au cours du cycle et ceci d'autant mieux que l'écart Zeeman des raies optiques est petit devant la largeur naturelle du niveau excité.

2. Les signaux de détection optique : absorption * ou fluorescence, s'expriment entièrement en fonction des éléments de la matrice densité dans l'état fondamental. Les expressions exactes de ces signaux sont établies.

3. Des déplacements de fréquence des raies de résonance magnétique peuvent être provoqués par l'excitation optique. Le mécanisme de ces déplacements relève de 2 processus physiques entièrement différents : La self-énergie des sous-niveaux Zeeman en présence du rayonnement excitateur qui peut différer d'un sous-niveau à l'autre; la conservation de la cohérence au cours du cycle de pompage qui permet de ramener dans l'effet Zeeman de l'état fondamental une partie de l'effet Zeeman de l'état excité.

\section{APPENDICE I \\ JUSTIFICATION DE L'APPROXIMATION SECULAIRE}

$$
\text { (cf. } \S_{-} I V-2 \text { ) }
$$

1. - Pour simplifier, considérons d'abord l'équation

$$
\mathrm{d} x / \mathrm{d} t=\left(a+b \mathrm{e}^{i \omega_{\mathrm{t}} t}\right) x .
$$


Są solution rigoureuse est :

$$
x=x_{0} \mathrm{e}^{a t+\frac{b}{i \omega_{\mathrm{f}}} \mathrm{e}^{i \omega_{\mathrm{f}} t}}
$$

où $x_{0}$ est une constante.

On peut écrire :

$$
x=x_{0} \mathrm{e}^{a t} \sum_{n=0}^{\infty}\left(\frac{b}{i \omega \mathrm{f}}\right)^{n} \times \frac{1}{n !} \mathrm{e}^{i n \omega_{\mathrm{f}} t} .
$$

Si $|b| \omega_{\mathrm{f}} \mid \ll 1$, on peut limiter le développement à :

$$
x=x_{0} \mathrm{e}^{a t}
$$

qui est la solution de

$$
\mathrm{d} x / \mathrm{d} t=a x,
$$

obtenue en négligeant le terme oscillant dans (1).

Ce résultat peut être retrouvé autrement. On résout

$$
\mathrm{d} x / \mathrm{d} t=\left(a+\lambda b e^{i \omega_{\mathrm{f}} t}\right) x
$$

par un développement en puissances de $\lambda$ et on fait ensuite $\lambda=1$. A l'ordre 0 :

$$
\mathrm{d} x^{(0)} / \mathrm{d} t=a x^{(0)},
$$

d'où

$$
x^{(0)}=x_{0} \epsilon^{a t} .
$$

A l'ordre 1 :

$$
\mathrm{d} x^{(1)} / \mathrm{d} t=a x^{(1)}+b \mathrm{e}^{i \omega_{\mathrm{f}} t} x^{(0)},
$$

d'où

$$
x^{(1)}=x_{0} \frac{b}{i \omega_{\mathrm{f}}} \mathrm{e}^{i \omega_{\mathrm{f}} t \in t}
$$

etc... On retrouve ainsi tous les termes du développement (3), dont on ne garde que le $1^{\text {er }}$ (ordre 0) si $b / \omega_{\mathrm{t}} \ll 1$.

Physiquement, le $2^{\mathrm{e}}$ terme du second membre de (1) oscille trop rapidement par rapport à son amplitude pour avoir des effets notables.

2. - Le système (IV-1) s'écrit sous une forme générale

$\frac{\mathrm{d} X_{n}}{\mathrm{~d} t}=\sum_{m}\left(A_{n m}+B_{n m} \epsilon^{i \omega_{\mathrm{f}} t}+C_{n m} \mathrm{e}^{2 i \omega_{\mathrm{f}} t}+\ldots\right) X_{m}$.

les coefficients $A, B, C$, ont pour ordre de grandeur $1 / T_{p}$. On résoudra de même par un développement en puissances de $\lambda$ le système

$$
\begin{aligned}
\mathrm{d} X_{n} / \mathrm{d} t=\sum_{m} A_{n m} X_{m}+\lambda \sum\left(B_{n m} \mathrm{e}^{i \omega_{\mathrm{f}} t}\right. & \\
& \left.+C_{n m} \mathrm{e}^{2 i \omega_{\mathrm{f}} t}+\ldots\right) X_{m} .
\end{aligned}
$$

Le système

$$
\mathrm{d} X_{n}^{(0)} / \mathrm{d} t=\sum_{m} A_{n m} X_{m}^{(0)}
$$

obtenu à l'ordre 0 n'est autre que celui de l' «appro- ximation séculaire ». Sa.solution générale est de la forme :

$$
X_{n}^{(0)}=\sum_{r} K_{r} \alpha_{r n} \mathrm{e}^{\beta_{r} t}
$$

où les $\alpha_{n r}$ et les $\beta_{r}$ sont des constantes dépendant des $A_{n m}$, les $K_{r}$ des constantes déterminées par les conditions initiales. Les $\beta_{r}$ ont pour ordre de grandeur $1 / T_{p}$. On peut toujours supposer les $\alpha_{n r}$ d'ordre de grandeur unité par une normalisation convenable. A l'ordre (1) on obtient :

$$
\begin{aligned}
\frac{\mathrm{d} X_{n}^{(1)}}{\mathrm{d} t}=\sum_{m} A_{n m} X_{m}^{(1)}+\sum_{m} & \left(B_{n_{m}} \mathrm{e}^{i \omega_{\mathrm{f}} t}\right. \\
& \left.\quad+C_{n_{m}} \mathrm{e}^{2 i \omega_{\mathrm{f}} t}+\ldots\right) X_{m}^{(0)} .
\end{aligned}
$$

On en déduit pour les $X_{n}^{(1)}$ des solutions de la forme :

$$
X_{n l}^{(1)}=\sum_{r s k} \alpha_{n r} \epsilon^{\beta} t \int_{0}^{t} M_{r s}^{k} \mathrm{e}^{\left[\left(\beta_{s}-\beta_{r}\right)+k i \omega_{t}\right] t^{\prime}} \mathrm{d} t^{\prime}
$$

où les $M_{r s}^{k}$ ont pour ordre de grandeur $K / T_{p}$ ( $K$ : ordre de grandeur de $K_{r}$ ), $k$ étant un nombre entier. On voit que le rapport des ordres de grandeur de $X_{n}^{(1)}$ et $X_{n}^{(0)}$ est $\frac{1}{T_{p}\left|\beta_{r}-\beta_{s}+i k \omega_{\mathrm{f}}\right|}$

Il en sera de même pour les rapports des termes d'ordre supérieur, $X_{n}^{(2)}$ à $X_{n}^{(1)} \ldots X_{n}^{(q+1)}$ à $X_{n}^{(q)}$ Si $\omega_{\mathrm{f}} T_{p} \gg 1$, on pourra se limiter aux ordres les plus bas, et en pratique on fera une erreur d'ordre de grandeur $1 / \omega_{\mathrm{f}} T_{p}$ en ne gardant que les termes d'ordre 0 (approximation séculaire).

\section{APPENDICE II}

Nous voulons démontrer que les valeurs propres de la matrice de relaxation $A_{m n}$ définie dans le précédent appendice et associée au système séculaire (IV-2), ont leur partie réelle négative ou nulle.

D'après la remarque faite dans (IV, B, 1) cette matrice se subdivise en plusieurs sous-matrices correspondant aux différentes valeurs de $\mu-\mu^{\prime}$. En particulier pour $\mu-\mu^{\prime}=0$, nous obtenons la matrice de pompage optique :

$$
\mathrm{d}_{\kappa \mu \mu} / \mathrm{d} t=-\left(\sum_{\mu^{\prime}} P_{\mu \rightarrow \mu^{\prime}}\right) f_{\mu \mu}+\sum_{\mu^{\prime}} P_{\mu^{\prime} \rightarrow \mu} f_{\mu^{\prime} \mu^{\prime}} .
$$

Montrons tout d'abord que les valeurs propres de la matrice de pompage optique ont leur partie réelle négative ou nulle.

Nous devons démontrer qu'aucune des quantités $\rho_{\mu \mu}(t)$ ne tend en module vers l'infini quand $t$ tend vers l'infini.

A l'instant initial $t=t_{0}$ toutes les quantités $p_{\mu \mu}\left(t_{0}\right)$ sont positives ou nulles (populations). D'autre part, à partir de (1), on établit facilement que

$$
\sum_{\mu} \frac{d}{d} \rho_{\mu \mu}=\sum_{\mu \mu^{\prime}}\left(-P_{\mu \rightarrow \mu^{\prime}}+P_{\mu \rightarrow \mu^{\prime}}\right) \rho_{\mu \mu}=0 .
$$


Si nous démontrons que les $\rho_{\mu \mu}(t)$ restent toujours positifs ou nuls au cours du temps, comme leur somme est constante, nous aurons démontré qu'ils ne peuvent tendre vers l'in fini.

Désignons par $t_{1}$ le premier instant après $t_{0}$ où l'une des quantités $p_{\mu \mu}$ (supposées toutes initialement positives) s'annule : $p_{\mu \mu}\left(t_{1}\right)=0$. Il s'ensuit d'après (1) que

$$
\left[\frac{\mathrm{d}}{\mathrm{d} t} \mathrm{~F}_{\mu \mu \mu}\right]_{t=t_{1}}=\sum_{\mu^{\prime} \neq \mu}\left(P_{\mu^{\prime}} \rightarrow \mu^{\prime}\right) \rho_{\mu^{\prime} \mu^{\prime}}\left(t_{1}\right) .
$$

Comme les $\rho_{\mu^{\prime} \mu^{\prime}}\left(t_{1}\right)$ sont positifs par hypothèse ainsi que les $P_{\mu^{\prime} \rightarrow \mu}$ d'après (IV-7) on en déduit que

$$
\left[\frac{\mathrm{d}}{\mathrm{d} t}[\mu \mu]_{t=t_{1}} \geqslant 0 .\right.
$$

$P_{\mu \mu}$ ne pourra donc jamais devenir strictement négatif. Cette démonstration se généralise facilement au cas où plusieurs $p_{\mu \mu}$ s'annulent en même temps pour la première fois.

Pour une valeur bien déterminée et non nulle de $\mu-\mu^{\prime}$, la matrice $A_{m n}$ est la matrice d'élargissement.

D'après la définition même des éléments d'une matrice densité, la solution rigoureuse des équations d'évolution $(\mathrm{I}, 9)$ satisfait à

$$
\left|\kappa \mu \mu^{\prime}(t)\right| \leqslant V \overline{\mid f \mu \mu, t) \quad f \mu^{\prime} \mu^{\prime}(t) \mid} .
$$

Mais nous avons démontré que moyennant les conditions (IV-3) et (IV-4) la solution rigoureuse de (I-9) était très voisine de celle des équations séculaires (IV-2), l'erreur relative étant de l'ordre de $1 / \omega_{\mathrm{f}} T_{p}$. L'inégalité (2) est donc valable également pour la solution de (IV-2). Comme nous venons de démontrer que les $p_{\mu \mu}(t)$ ne tendent pas vers l'infini, il s'ensuit qu'il en est de même pour les $\left|\rho_{\mu \mu^{\prime}}\right|$ et que les valeurs própres 'des matrices d'élargissement ont leur partie réelle négative ou nulle.

Démontrons enfin que dans le cas de 2 niveaux ( $\S \mathrm{IV}, \mathrm{B}, 3$ ), la quantité $1 / T_{2}^{\prime \prime}$ est positive. Physiquement ceci signifie que dans le cas de 2 niveaux, il y a une "restitution " partielle de la cohérence au cours de la retombée et non une destruction supplémentaire

$$
\frac{1}{T_{2}^{\prime \prime}}=\frac{B_{\frac{1}{2}-\frac{1}{2}}^{\frac{1}{2}-\frac{1}{2}}}{T_{\mathrm{p}}} \frac{\Gamma^{2}}{\Gamma^{2}+\left(\omega_{\mathrm{e}}-\omega_{\mathrm{t}}\right)^{2}} .
$$

Il suffit de démontrer que $B_{\mu \mu^{\prime}}^{\mu \mu \prime^{\prime}}$ est positif.

$$
\begin{aligned}
& b_{\mu \mu^{\prime}}^{\mu \mu^{\prime}}=\sum_{\substack{m, m m^{\prime} \\
m-m^{\prime}=\mu-\mu^{\prime}}}\left\langle m_{1}^{\prime} \boldsymbol{e}_{\gamma_{0}} D \mid \dot{\mu}\right\rangle\left\langle\mu^{\prime}\left|\boldsymbol{e}_{\circ} D\right| m^{\prime}\right\rangle \\
& C_{1 I}(F, m ; m-\mu, \mu) C_{1 I}\left(F, m^{\prime} ; m^{\prime}-\mu^{\prime}, \mu^{\prime}\right) .
\end{aligned}
$$

Mais d'après l'expression (III-5) des $\left\langle\mu\left|e_{\lambda} D\right| m\right\rangle$, il vient en utilisant le fait que dans la sommation sur $m$ et $m^{\prime}, m-\mu=m^{\prime}-\mu^{\prime}$

$$
\begin{aligned}
I_{\mu \mu^{\prime}}^{\mu \prime^{\prime}}=\sum_{\substack{m, m^{\prime} \\
m-m^{\prime}=}}\left[C_{\mathbf{1}^{I}\left(F, \mu^{\prime}\right.}(F ; m-\mu, \mu)\right]^{2} & \\
& {\left.\left[C_{\mathbf{1}} I^{I} F, m^{\prime} ; m^{\prime}-\mu^{\prime}, \mu^{\prime}\right)\right]^{2} } \\
& |<\mu=0| \mathbf{e}_{\lambda} D\left|m_{J}=m-\mu>_{I=0}\right|^{2} \geqslant 0 .
\end{aligned}
$$

Manuscrit reçu le 20 février 1961.

\section{BIBLIOGRAPHIE}

[1] Barrat (J. P.) et Cohen-Tannoudi (C.), J. Physique Rad., 1961, $22,329$.

[2] Abragam (A.), Principes of nuclear magnetism, Oxford Press, ch. 8.

[3] Margerie (J.), Diplôme d'Études Supérieures, Paris, 1955. Cohen-Tannoudj (C.), Diplôme d'Études Supérieures, Paris, 1956. Cagnac (B.), Thèse, Paris, 1960.
[4] Mitchell et Zemansky, Resonance radiation and excited atoms, $\cdot$ ch. 5 .

[5] Cohen-Tannoudi (C.), C. R. Acad. Sc., 1961, 252, 394.

[6] Ayant (Y.), J. Physique Rad., 1955, 16, 411.

[7] Bell (W. E.) et Bloом (A. L.), Phys. Rev., 1957, 107, 1559 . 